\title{
Recommendations of procedures to follow in the case of ovarian lesions in girls
}

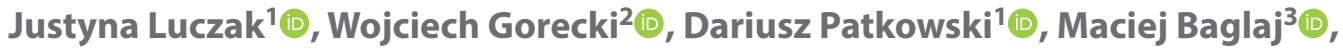 \\ Agnieszka Drosdzol-Cop ${ }^{4}\left({ }^{-}\right.$, Elzbieta Adamkiewicz-Drozynska ${ }^{5}{ }^{-}$,

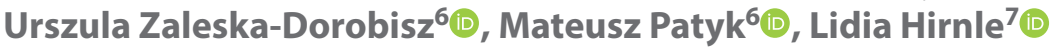 \\ ${ }^{1}$ Pediatric Surgery and Urology Department, Wroclaw Medical University, Wroclaw, Poland \\ ${ }^{2}$ Department of Pediatric Surgery, Jagiellonian University Medical College, Cracow, Poland \\ ${ }^{3}$ Department of Propedeutic of Pediatrics and Rare Diseases, Wroclaw Medical University,Wroclaw, Poland \\ ${ }^{4}$ Chair and Clinical Department of Gynecology, Obstetrics and Gynecological Oncology, Medical University of Silesia, \\ Katowice, Poland \\ ${ }^{5}$ Medical University of Gdansk, Poland \\ ${ }^{6}$ Department of General and Pediatric Radiology, Wroclaw Medical University, Wroclaw, Poland \\ ${ }^{7} 1^{\text {st }}$ Department and Clinic of Gynaecology and Obstetrics, Wroclaw Medical University, Wroclaw, Poland
}

\begin{abstract}
This study presents current recommendations of the Polish Association of Pediatric Surgeons (PTChD) regarding diagnostics and treatment of ovarian lesions in girls. They are based on many years of the authors' clinical experience as well as a review of international literature and include practical clinical guidelines. The recommendations were formulated in cooperation with the Polish Association of Pediatric Oncology and Hematology (PTOHD), Polish Pediatric and Adolescent Gynecology Section of the Polish Society of Gynecologists and Obstetricians (PTG) and Polish Pediatric Section of the Polish Society of Radiology (PLTR). Only better understanding of prepubertal ovarian biology and natural history of its pathology may help to introduce efficient and safe diagnostic and therapeutic strategies for girls. The prepared document has been supplemented with treatment algorithms.
\end{abstract}

Key words: ovarian neoplasms; ovarian masses; child; management; algorithms

Ginekologia Polska 2022; 93, 1: 76-87

\section{INTRODUCTION}

This study presents current recommendations of the Polish Association of Pediatric Surgeons (PTChD) regarding diagnostics and treatment of ovarian lesions in girls. They are based on many years of the authors' clinical experience as well as a review of international literature and include practical clinical guidelines. The recommendations were formulated in cooperation with the Polish Association of Pediatric Oncology and Hematology (PTOHD), Polish Pediatric and Adolescent Gynecology Section of the Polish Society of Gynecologists and Obstetricians (PTG) and Polish Pediatric Section of the Polish Society of Radiology (PLTR). The guidelines can only be useful in clinical practice and cannot be regarded as ultimate therapeutic recommendations. Followed by new clinical experience, they will be subject to modifications (validation every 3-5 years) aimed at attaining the most efficient therapeutic effect possible while maintaining the highest level of safety and keeping side effects and complications to a minimum. The prepared document has been supplemented with treatment algorithms. We expect these guidelines to be helpful in detecting malignancy, in preserving more ovarian tissue during surgery of ovarian lesions and torsions and in reducing the rate of surgical management of noncomplicated ovarian cysts.

\footnotetext{
Corresponding author:

Justyna Luczak

Pediatric Surgery and Urology Department, Wroclaw Medical University, 213 Borowska St, 50-556 Wroclaw, Poland

e-mail: sitnikjustyna@gmail.com
} 


\section{EPIDEMIOLOGY OF ADNEXAL MASSES}

\section{Functional ovarian lesions [1-4]}

Types:

a) Follicular cyst

b) Hemorrhagic follicular cyst

c) Corpus luteum

d) Corpus luteum cyst

e) Hemorrhagic corpus luteum cyst

Adnexal functional lesions are the most frequent reason for ovarian "tumors" in children. According to relevant publications, they constitute from $17 \%$ to $44 \%$ of all ovarian pathologies in children. Functional lesions may be discovered accidentally or in an emergency room due to experienced pain. Imaging tests usually reveal a simple cyst, which is small in size $(1-3 \mathrm{~cm})$. The cysts can also grow larger (> $5 \mathrm{~cm}$ ) and cause acute pain because of rupture or adnexal torsion. Data concerning the incidence of functional lesions in children are limited. Nevertheless, it is known that they occur more often and reach larger sizes in the peripubertal period due to an increase in gonadotropic stimulation. In infants, they occur as a result of hormonal stimulation by the mother's organism in the fetal period. According to several available publications, the incidence of these lesions oscillates between $2 \%$ and $5 \%$ in the period between 0 and 9 years of age and between $4 \%$ and $32 \%$ in the period between 10-18 years of age.

It should be noted that follicular cysts may also produce estrogens and cause symptoms of precocious puberty (peripheral precocious puberty). Such lesions are usually self-limiting and do not require treatment. In order to differentiate them from other reasons for precocious puberty (hormonally active tumors, McCune-Albright syndrome, central cause), a series of tests should be performed taking into consideration physical examination, bone age measurement, estradiol level measurement, GnRH (gonadotropin-releasing hormone) stimulation test (gold standard) as well as imaging tests (ultrasound, possibly also MRI - magnetic resonance imaging). After eliminating other causes of precocious puberty, surgical treatment is only advisable in the event of cyst torsion or development of secondary sex characteristics, but also when the cyst does not disappear within three months.

Ovarian endometrial cysts ("chocolate cyst") is a benign estrogen-dependent cyst occurring in women in the reproductive period. It affects $17-44 \%$ of women suffering from endometriosis. The incidence of the lesions in girls before and after menarche is extremely rare (single published descriptions of cases). A non-surgical treatment is possible $[3,4]$

\section{Ovarian tumors [1, 3-11]}

Ovarian tumors can derive from three cell lines: epithelial tissue cells, ovarian stroma cells and pluripotent germ cells. In children, most ovarian tumors (> 70\%) originate from germ cells: these are mostly benign mature teratomas. In patients over 18 years of age, a vast majority of tumors come from the epithelial tissue: benign adenomas and cancers constitute $>60 \%$ of all ovarian tumors and $80-90 \%$ of malignant neoplasms. The classification presented below is based on WHO's (World Health Organization) classification and includes lesions occurring in children.

\section{Benign tumors \\ Germ cell tumors}

a) Teratoma maturum (mature teratoma) is the most common neoplastic ovarian tumor in children. These neoplasms are composed of cells that are derivatives of all the three germ layers. A special type of a mature teratoma is a dermoid cyst, which is made of one germ layer (monodermal). Most publications state that the peak incidence of these lesions is in the early teenage years. The exact incidence remains unknown. Mature teratomas constitute approximately $50 \%$ of all ovarian tumors in children and often occur bilaterally (10-15\%). Clinically, mature teratomas are most often slow-growing cystic structures that demonstrate characteristic features in an ultrasound examination.

b) Teratoma immaturum (immature teratoma) may macroscopically resemble a mature teratoma and is difficult to classify histologically. The key difference between the two types of teratoma is the presence of immature (germinal) cells, mainly of neurogenic origin, in the immature one. Measurements of tissue quantity allow to classify their maturity level. They occur much less frequently than mature teratomas. It is difficult to specify their exact incidence since these lesions are often misclassified. It was believed that these tumors have a tendency to demonstrate features of "local malignancy". However, the newest publications assume that, in their pure form, these lesions are benign tumors. The essence is to understand the natural course of development of an immature teratoma. Removed completely and well-assessed histologically, as a histopathology specimen, it is a benign tumor. Frequent coincidence of small malignant tumor foci (most often as a yolk sac tumor that can be misdiagnosed as Heifetz lesions, which frequently occur in teratomas) may be responsible for its "malignant" clinical course with distant metastases. These cases are described in the literature as metastatic immature teratomas. Serum AFP (alpha-fetoprotein) cut-off level of $1000 \mathrm{ng} / \mathrm{mL}$ is used as one of the indicators of the presence of malignant elements. Higher values usually indicate the presence of a malignant tumor. According to the present state of knowledge, immature teratomas in their pure form require surgical treatment only. Nevertheless, a reliable histopathological test including grading is key to post- 
operative management. Consultation with an oncologist is indicated.

\section{Stromal tumors}

a) Thecoma - produces estrogen, very rarely develops in children

b) Fibroma - very rarely diagnosed in children

\section{Epithelial tumors}

Epithelial tumors - typical in adults, rarely develop in children, however, the exact epidemiological data are not known.

a) Cystadenoma - serous or mucinous, usually cystic or complex masses in imaging tests. They can reach enormous sizes, occupy the entire abdominal cavity, exert pressure on organs in the chest and deform costal arches.

\section{Malignant tumors}

The exact epidemiological data for Poland are not known. In one American study of 2008, which involved 1037 children diagnosed with a malignant ovarian tumor, the incidence of this tumor was calculated. The age-adjusted incidence of malignant ovarian tumors in children amounted to 0.102 for children before the age of nine and 1.072 for children between 10 and 19 years of age per 100.000 per year. The authors of the majority of available publications agree that the incidence of malignant lesions is low. However, the characteristics of the patients' age is a disputable issue. Nevertheless, numerous publications emphasize the rarity of incidence of these lesions in very young children: girls before the age of four constituted a mere $1.5 \%$ of all the patients involved in the above study.

\section{Germinal tumors}

Germinal tumors - most frequent malignant tumors in children. According to the literature, they constitute between 60 and $80 \%$ of all malignant ovarian tumors. All malignant germ cell tumors in advanced stages require chemotherapy. However, the management in stage I/II depends on each individual case. In lower stages, the management is chosen by an oncological team based on the analysis of recurrence risk factors and the current treatment protocols. Recently created Malignant Germ Cell Tumor International Collaborative in its publications gathering data from four clinical trials recommends surgery alone for patients with stage I/II, grade 1/2 tumors and suggests conducting of a prospective trial of observation after surgery for patients with grade $2 / 3$, stage II-IV tumors. This approach might be encouraged by the results of Park et al., as they revealed that most stage I malignant ovarian germ cell tumor recurrences can be successfully salvaged by surgery and BEP chemotherapy without compromising the overall survival. a) Dysgerminoma - histologically, it is the equivalent of a germ cell tumor of the testicle (seminoma). It is most frequently diagnosed in teenage patients. Bilateral occurrence of this type of tumor is found in 5 to $20 \%$ of patients. The presence of multinuclear syncytiotrophoblastic giant cells is frequently associated with elevated LDH (lactate dehydrogenase) levels and rarely with elevated $\beta$ hCG ( $\beta$-subunit of human chorionic gonadotropin) levels.

b) Tumor sinus endodermalis/yolk sac tumor - produces the AFP marker.

c) Carcinoma embryonale (embryonal carcinoma) - usually occurs as part of a mixed tumor (hence the wrong assumption in the past that it produced $\beta$ hCG and AFP or was endocrinologically active and caused precocious puberty by producing estrogens).

d) Choriocarcinoma - produces $\beta$ hCG.

e) Gonadoblastoma - neoplastic tumor that forms most frequently in dysgenetic gonads in people with disorders of sex development.

f) Tumor germinalis mixtus malignus (malignant mixed germ cell tumor) - produces AFP (due to yolk sac tumor component).

\section{Stromal tumors}

Stromal tumors - constitute $6-17 \%$. They originate from primary undifferentiated mesenchymal cells that possess potential abilities to differentiate in the direction of granulosa cells tumor and theca cells within the female gonad and in the direction of Leydig and Sertoli cells within a testicle. They are, therefore, hormonally active and may lead to precocious puberty or virilization.

a) Tumor granulocellularis typus juvenilis (juvenile granulosa cell tumor) - the most frequent in the group of malignant stromal tumors. Its level of aggressiveness differs depending on the details of the histological structure. Advanced forms require adjuvant chemotherapy.

b) Androblastoma (Sertoli-Leydig cell tumor) — produces estrogen or testosterone, advanced forms require adjuvant chemotherapy.

c) Gynandroblastoma - very rare.

\section{Epithelial neoplasms}

Epithelial neoplasms - constitute up to $2 \%$ of all malignant ovarian lesions. More frequently develop in older girls (their incidence increases after 14 year of age). Most of these tumors require adjuvant chemotherapy.

Cystadenocarcinoma (ovarian cancer) — serous or mucinous.

\section{Other malignant neoplasms:}

a) Epithelial tumor - borderline malignancy

b) Polyembryoma 
c) Metastases and infiltrations of another neoplasm (e.g., lymphoma)

\section{DIAGNOSTIC METHODS}

Imaging tests and symptoms - see: TREATMENT ALGORITHMS (Fig. 1-6) [4, 6, 8, 12-19]

The goal of diagnostic procedures for ovarian tumors in children is to establish whether there is a risk that the lesion is of malignant nature, assess the possibility of fertility-sparing therapy if the lesion proves to be benign and verify the necessity of surgery in the case of a non-cancerous lesion. During the process, we recommend following algorithms that take presented symptoms (acute or mild), imaging results (basic ultrasound test) and evaluation of serum tumor marker concentration (depending on indications) into consideration. Based on the recent knowledge we have prepared separate algorithms for an ultrasound test result that includes Ueland Index (MI, morphology index - an ultrasound-based scoring system that include the tumor volume along with its structural appearance)

\section{ALGORITHM WITHOUT ACUTE SYMPTOMS (WITHOUT UELAND INDEX AND OCS* EVALUATION)}

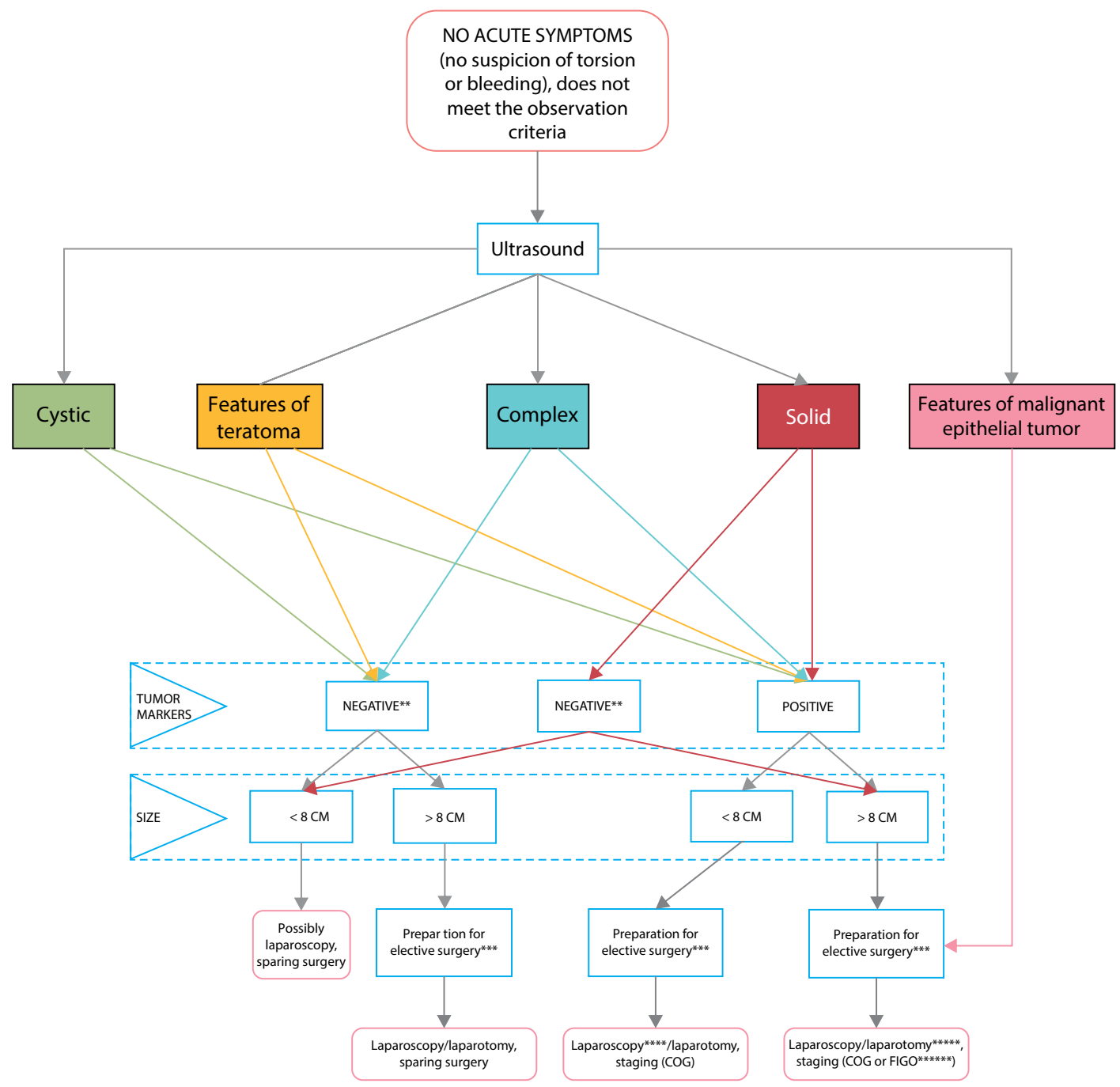

*OCS - OVARIAN CRESCENT SIGN, Ueland Index (morphology index, MI) - an ultrasound-based scoring system that include the tumor volume along with its structural appearance.

**A-125 - might be slightly elevated; in girls over the age of 12, it is advisable to widen the diagnostic spectrum to include the risk of ovarian malignancy algorithm (ROMA) as well as the risk of malignancy index (RMI) modified for children; in children up to 1 year of age at least two evaluations of AFP level every 2 weeks (consider elevated only if the level is rising); in the case of elevated $\beta$-hCG in sexually active girls always exclude pregnancy

*** includes tumor markers evaluation

**** laparoscopy also for radical resection

***** laparoscopy - only as a part of STAGING procedure

***** Children's Oncology Group; International Federation of Gynecology and Obstetrics (for a a high possibility of malignancy that may be of epithelial nature)

Figure 1. Algorithm — without acute symptoms (without Ueland index and OCS evaluation) 




*OCS - OVARIAN CRESCENT SIGN, Ueland Index (Morphology Index) - an ultrasound-based scoring system that Include the tumor volume along with Its structural appearance. ** includes tumor markers evaluation *** CA-125 - might be slightly elevated; in girls over the age of 12 , it is advisable to widen the diagnostic spectrum to include the risk of ovarian malignancy algorithm (ROMA) as well as the risk of malignancy Index (RMI) modified for children; in children up to 1 year of age at least two evaluations of AFP level every 2 weeks (consider elevated only if the level is rising); in the case of elevated $\beta$-hCG in sexually active girls always exclude pregnancy **** laparoscopy - as a part of STAGING procedure ***** Children's Oncology Group; International Federation of Gynecology and Obstetrics (for a a high possibility of malignancy laparoscopy - as a part of STAGING
that may be of epithelial nature)

Figure 2. Algorithm — without acute symptoms (with Ueland index and OCS evaluation)

and ovarian crescent sign evaluation. A separate situation is also an accidental discovery of an ovarian lesion during a procedure performed for other indications. An additional file shows a detailed description of the imaging tests useful in the diagnosis of ovarian lesion (see Supplement 1).

\section{Tumor markers [4, 6, 8, 12, 15-16, 20-23]}

Due to the lack of randomized studies analyzing children, it is advisable to measure the below-mentioned serum markers when a lesion is first noticed in an imaging test in patients with a solid lesion of any size, a mixed (solid and cystic) lesion with a diameter over $5 \mathrm{~cm}$ or a cystic lesion larger than $8 \mathrm{~cm}$. In other cases, the evaluation of markers may be performed during the next ultrasound test.
Markers: AFP (alpha-fetoprotein), $\beta$ hCG ( $\beta$-subunit of human chorionic gonadotrophin), LDH (lactate dehydrogenase), CA125 (cancer antigen CA-125) and, should it be possible in a given surgical center, estradiol, testosterone and inhibin $A$.

In newborns and infants, it is recommended to perform the $\beta$ hCG and AFP (at least two evaluations tests every two weeks. A gradual decrease in marker concentration indicates a non-cancerous character of a lesion.

In girls over the age of 12 , it is advisable to widen the diagnostic spectrum to include the risk of ovarian malignancy algorithm (ROMA) as well as the risk of malignancy index (RMI) modified for children. CA-125 might be slightly elevated even in non-malignant lesions. 


\section{ALGORITHM — ACUTE SYMPTOMS (WITHOUT UELAND INDEX* EVALUATION)}

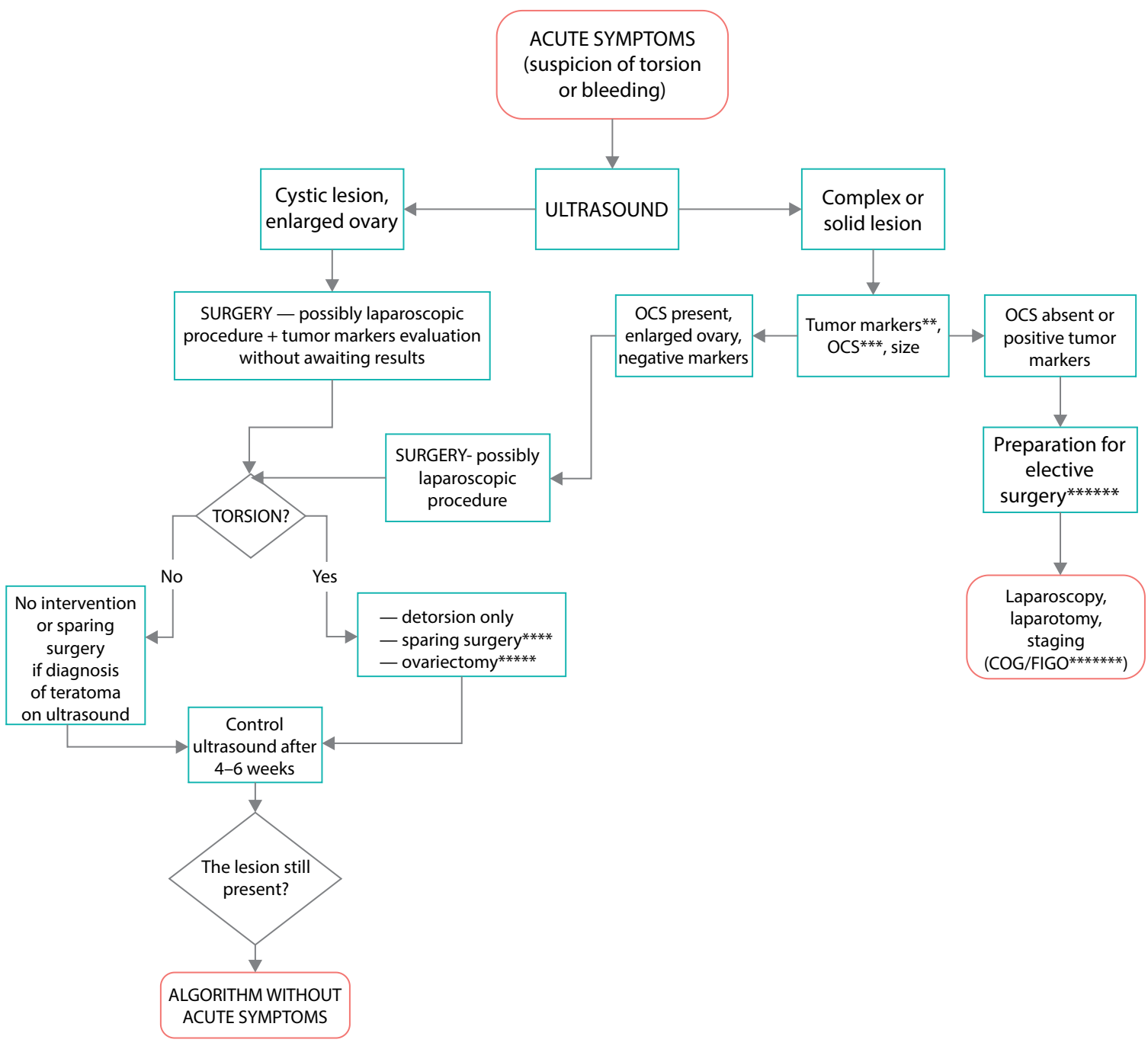

*Ueland Index (Morphology Index) - an ultrasound-based scoring system that include the tumor volume along with its structural appearance.

**CA-125 - might be slightly elevated; in girls over the age of 12 , it is advisable to widen the diagnostic spectrum to include the risk of ovarian malignancy algorithm (ROMA) as well as the rink of malignancy index (RMI) modified for children; in children up to 1 year of age at least two evaluations of AFP level every 2 weeks (consider elevated only If the level is rising): in the case of elevated $\beta$-hCG in sexually active girls always exclude pregnancy ***OVARIAN CRESCENT SIGN

****sparing surgery - after detorsion, possible use of Intraoperative ultrasound/ also In case of teratoma features on preoperative ultrasound ***** ovariectomy - only in case of autoamputation or advanced necrosis

********includes tumor markers evaluation

******includes tumor markers evaluation (for a high possibility of malignancy that may be of epithelial nature)

Figure 3. Algorithm — acute symptoms (without Ueland index evaluation)

In the case of elevated $\beta$ hCG in sexually active girls, pregnancy should be always excluded. In the case of elevated LDH level a lymphoma should be excluded.

TREATMENT METHODS [1, 4, 6, 8, 12, 24-26]

\section{Observation (see also: TREATMENT ALGORITHMS - Figure 6)}

Possible in the case of functional lesions. The treatment plan should result from a risk-benefit ratio analysis:

\section{Observation choice criteria} In everyone: certainty that the lesion is of ovarian origin Newborn:

a) Any asymptomatic lesion regardless of an ultrasound test Infant:

a) Lesions observed since the neonatal period (no progression of the lesion, decrease in marker level)

b) Asymptomatic lesions (in practice $<5-6 \mathrm{~cm}$ )

c) Simple and complex cysts 
ALGORITHM — ACUTE SYMPTOMS (WITH ULEAND INDEX AND OCS* EVALUATION)

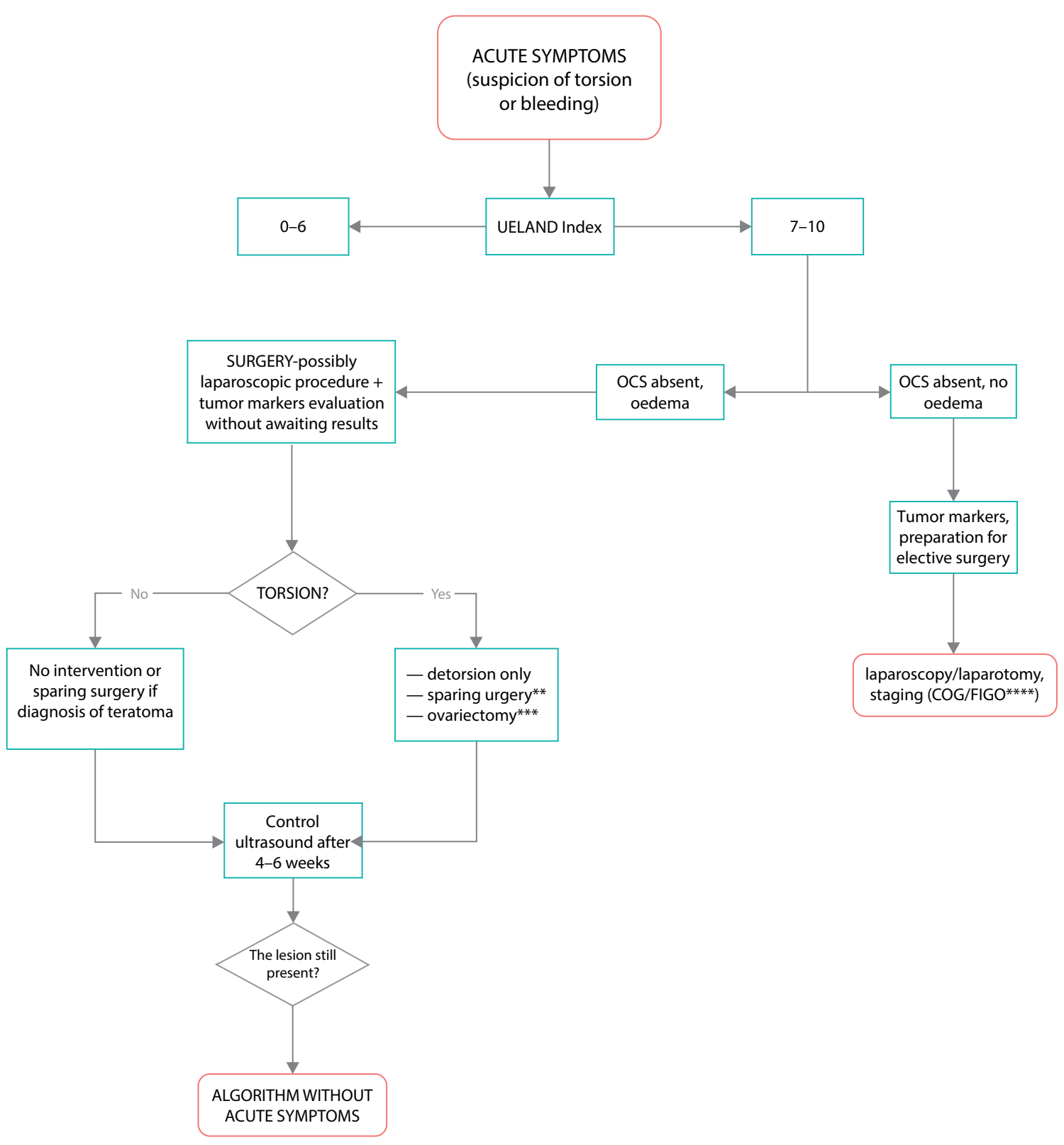

*OCS - OVARIAN CRESCENT SIGN, Ueland Index (Morphology Index) - an ultrasound-based scoring system that include the tumor volume along with its structural appearance.

**sparing surgery - after detorsion, possible use of intraoperative ultrasound/ also in case of teratoma features on preoperative ultrasound

*** ovariectorny - only in case of autoamputation or advanced necrosis

****laparoscopy only as a part of STAGING procedure; Children's Oncology Group; International Federation of Gynecology and Obstetrics

(for a high possibility of malignancy that may be of epithelial nature)

Figure 4. Algorithm — acute symptoms (with Ueland index and OCS evaluation)

Older child:

d) No acute abdominal symptoms, no additional symptoms (no precocious puberty, virilization, general symptoms, mass effect)

e) Size up to $8 \mathrm{~cm}$

f) Simple and complex cysts
Procedures during observation Newborns and infants:

a) Ultrasound test after 4 weeks

b) Monitoring marker concentration: $\beta$ hCG, AFP, estradiol (minimum 2 markers)

Older children: 


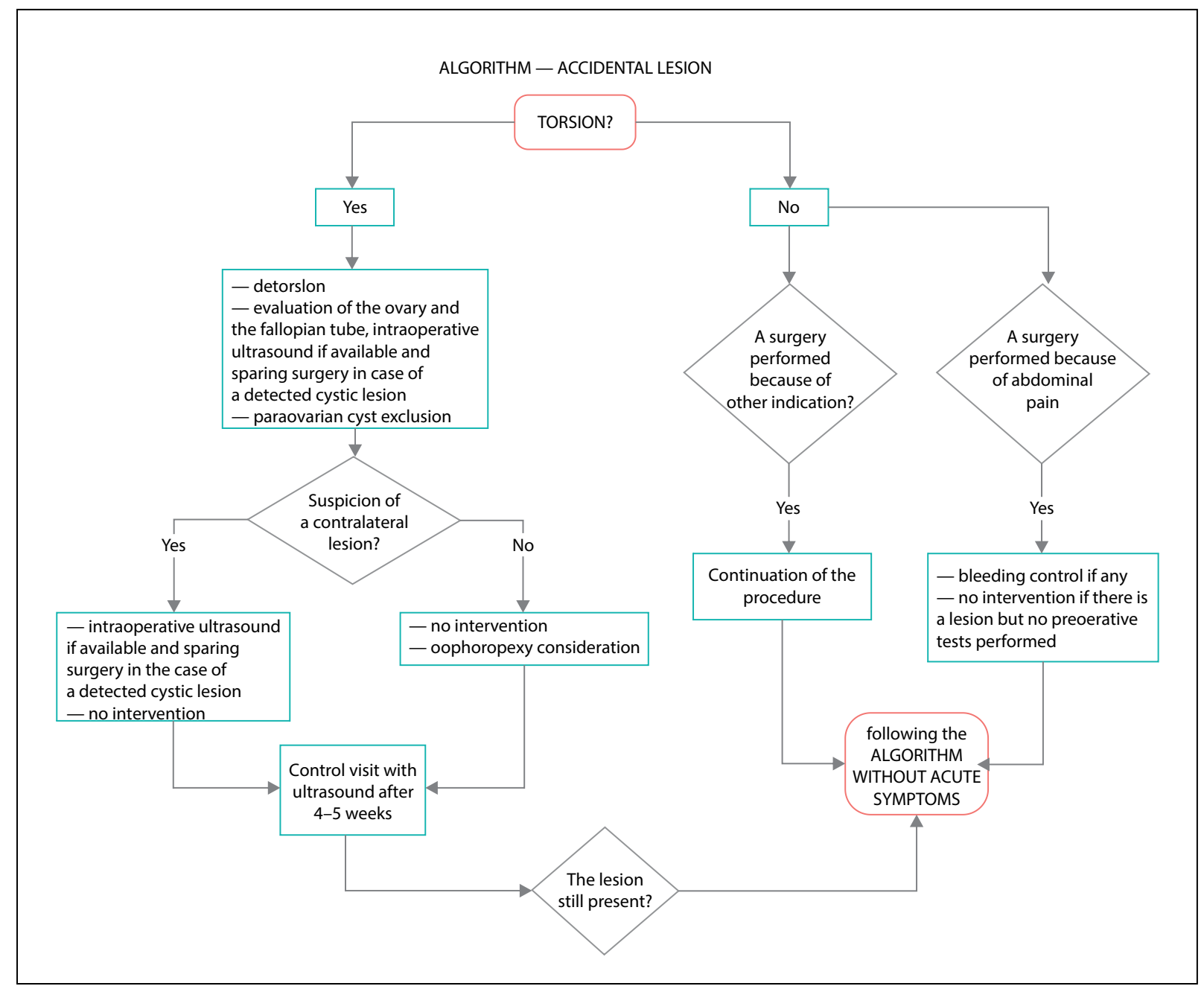

Figure 5. Algorithm - accidental lesion

a) Information about a possibility of torsion

b) Ultrasound test after menstruation for 3-6 consecutive cycles or after four weeks in prepubertal patients (assessment of changes in size and character)

c) Marker concentration evaluation (AFP, $\beta$ hCG, CA-125, ROMA > 12 years of age) - in the case of a solid lesion, complex lesion with a diameter over $5 \mathrm{~cm}$ or a cystic lesion larger than $8 \mathrm{~cm}$, a blood test is performed after the lesion is identified in an imaging test. In other cases, a blood test may be performed at the control ultrasound test

d) Considering hormone therapy: utilizing progestogens, contraceptive therapy for at least three months and another follow-up. Sometimes, indication to include $\mathrm{GnRH} / \mathrm{Tamoxifen}$ analogues (in the case of symptoms of precocious puberty).

Should the lesion remain: qualification for surgical treatment (preparation for elective surgery):

a) Wait (3-6 months of observation) b) Take age, imaging tests (the lesion's size and echogenicity, visible ovarian tissue), previous surgeries (tendency to frequently develop functional lesions in the remaining ovary after ovariectomy) into consideration

c) Always repeat an ultrasound of the abdomen/pelvis and tumor markers evaluation before surgery

d) In case of diagnostic uncertainty, consider CT (computed tomography) or MRI

e) Before surgery perform an anti-Müllerian hormone $(\mathrm{AMH})$ test and a follicle-stimulating hormone (FSH) test or secure a blood serum sample (freeze) in order to perform the tests at a later time.

\section{Laparoscopy}

Laparoscopy is currently the method of choice in the case of lesions without malignant features in preoperative tests (see:TREATMENT ALGORITHMS — Figures 1-6). Endoscopy allows to assess the size of a lesion and its safe enuclea- 


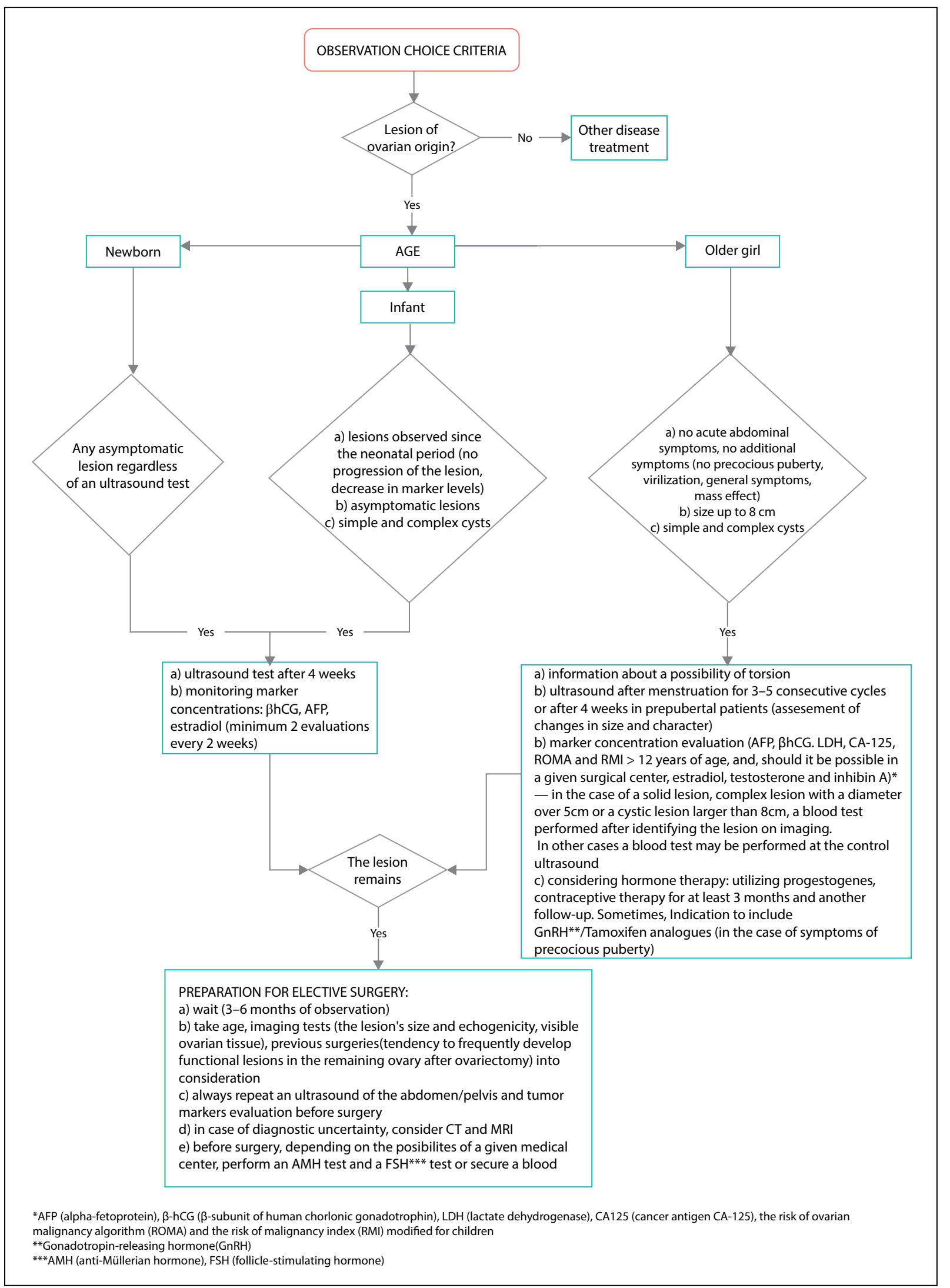

Figure 6. Algorithm — observation 
tion or marsupialization. It is also an excellent diagnostic tool (including exclusion of ovarian torsion and making the final deciding concerning the surgical technique) and proves useful in the removal of benign lesions, including the most common malignant tumor, i.e., mature teratoma. However, in the case of lesions suspected of malignancy, laparoscopic resection has not been advised so far. Therefore, the assessment of the risk of malignant lesion incidence is the most difficult decisive element in the process of selecting the right therapeutic procedure. No randomized study that would allow to objectively choose the proper malignancy indicators of ovarian lesions has been conducted so far. Still, one of the goals of this study is to improve the efficiency of malignant lesion diagnoses based on the available knowledge. A large size of a lesion is one of the malignancy risk indicators. It poses the most significant obstacle in the application of the laparoscopic technique in the case of solid lesions. However, it has been proven that in the case of tumors smaller that $7 \mathrm{~cm}$ in diameter there is no increased risk of damaging their capsule during laparoscopy. That is why, it may soon be possible to demonstrate the safety of the application of endoscopy in the treatment of malignant ovarian tumors. As is the case with children, there have been no randomized studies on the use of laparoscopy in stage I malignant ovarian tumors conducted among adult patients either. Nevertheless, other studies suggest that the technique is efficient and does not pose danger to oncological principles. Moreover, ovarian cancer in adults is a tumor of higher malignancy than the majority of malignant neoplasms in children. The lack of randomized studies seems to be the only obstacle for laparoscopy to be applied in the resection of malignant ovarian lesions of smaller sizes, even though it can complement the treatment as its initialization performed in order to inspect the peritoneal cavity with consecutive conversion to minilaparotomy and a resection of the lesion performed in the open way, should it prove large. Properly performed staging guarantees the correct assessment of the disease's progression stage and the right choice of treatment to complement a surgical procedure and offers a possibility to make a decision not to perform the surgery at all. It is important to remember that the aim of the treatment is not only to save the patient's life but also to secure her future fertility in each possible case. The efficiency of the proposed algorithms and a possible reasonability to perform laparoscopy in the resection of malignant ovarian tumors need to be verified in multi-center prospective studies. In the case of a large benign lesion, should there be uncertainty as to the possibility of saving the ovarian tissue in laparoscopy, it is advisable to perform laparotomy by Pfannenstiel incision and remove the tumor from the abdominal cavity after decompression puncture in order to perform sparing surgery.

\section{Laparotomy}

This method is recommended when the presence of a malignant lesion is suspected. Should such a suspicion occur, it is necessary to perform staging. In the case of large benign lesions, it is advisable to perform laparotomy by Pfannenstiel incision and remove the tumor from the abdominal cavity after decompression puncture.

\section{Staging (assessment of tumor's progression stage)}

The current guidelines concerning the assessment of the disease's stage in the case of epithelial neoplasms in adults have been developed by the International Federation of Gynecology and Obstetrics (FIGO). The removal of the tumor together with an oviduct is accompanied by an assessment of the amount of fluid in the peritoneal cavity, its cytology and biopsies taken from the pelvic walls, the pouch of Douglas and the paracolic sulci area. The surface of the diaphragm should be evaluated by means of cytology or biopsy. Serous membrane of the intestine and the mesentery should also be evaluated and the greater omentum removed. Moreover, pelvic and aortic lymphadenectomy should be performed. This protocol allows to preserve the uterus and the contralateral ovary in young women in a lower stage who wish to maintain their reproductive potential.

The current procedures regarding the assessment of the disease's progression stage in the case of germ cell tumors of the ovary in children have been developed by the CHILDREN'S ONCOLOGY GROUP (COG). These guidelines include:

a) cytology of peritoneal cavity fluid or washing, assessment of the peritoneum with biopsy or removal of possible lesions;

b) palpatory examination of retroperitoneal lymph nodes with biopsy of the solid and enlarged ones;

c) inspection and palpation of the omentum and resection of abnormal parts;

d) inspection and palpation of the contralateral ovary in case of abnormalities;

e) resection of the affected ovary with possible preservation of the oviduct.

Due to positive results achieved in the treatment of patients diagnosed with stage I malignant germ cell tumor of the ovary even in the case of recurrence (and the application of chemotherapy in such case), proceeding in accordance with COG's guidelines seems highly justified. The treatment procedure in the case of malignant epithelial neoplasm or borderline malignancy is connected with more doubts. The few studies in the area of these neoplasms in children do not offer enough certainty to regard the COG's procedures as safe in their case. Nevertheless, they may be sufficient when lesions in their initial stages are concerned. An incom- 
plete assessment of the stage of progression in these cases requires close observation for recurrence.

\section{Conclusions}

Intraoperative identification of a malignant lesion may be very difficult. The first step is a preoperative malignancy risk evaluation of the lesion (see:TREATMENT ALGORITHMS — Figure 1-6). In the case of a high possibility of malignancy of the lesion, which is limited to a gonad, in girls, it is advisable to follow the procedures developed by COG. In the case of lesions with a high possibility of malignancy that may be of epithelial nature [imaging test result (see: Additional file 1), markers characteristic of epithelial lesions, older girls (> 12 years of age)], an expansion of progression stage evaluation advised by FIGO should be taken into consideration (as in the case of patients who wish to retain their reproductive potential).

\section{Scope of treatment}

In each possible case, one should aim at fertility-sparing treatment. According to the few studies of treatment results in pediatric surgery centers, the percentage of fertility-sparing surgeries in the case of benign lesions oscillates between $24 \%$ and $83 \%$ worldwide, which is a highly uneven distribution. Efforts should be made to make these results more balanced and increase the percentage of the saved gonads. It is also important to understand what exactly fertility-sparing treatment consists in (see below).

\section{Fertility-sparing treatment of ovarian tumors in children}

This procedure is based on sparing the healthy part of an ovary after resection of an intraovarian lesion (sparing the contralateral ovary after surgical removal of the whole ovary due to a tumor is not considered a sparing procedure!). One of its types is cystectomy, which is resection of an ovarian cyst, and tumorectomy, which is a surgical removal of a neoplasm that is not a cyst. A so-called bivalving can be used in order to enucleate small benign lesions of the ovary, whereas large lesions can be resected with a margin and enucleated or a stripping technique can be used instead.

\section{Important issues}

Before dissecting, the type of the lesion should be identified (paraovarian or intraovarian);

a) One should always try to enucleate cystadenomas;

b) Intraoperative perforation and puncture are not adverse outcome risk factors (in the case of cystic lesions and negative markers);

c) Size of the lesion is not a limitation;

d) Torsion is not a limitation; e) Long-time observation will prove whether these patients are more prone to cancer recurrence/development.

Cyst fenestration is the least invasive procedure. It can be performed when the follicular cyst has been identified as the reason for the symptoms (the lesion should not be dissected when it has been accidentally discovered during surgery performed due to other indications). Bilateral and recurring lesions occur in $8-20 \%$ of patients. Insufficient caution in determining indications for operative treatment may result in multiple interventions, loss of the ovary and, in extreme cases, castration. One should also remember about the option of puncture in the case of large or even giant lesions with a low-level risk of malignancy, which offers a possibility of an easy and minimally invasive removal of even very large lesions. A lesion can also be placed in an endobag and sucked out of the peritoneal cavity after exteriorizing the endobag through the umbilical incision. In case of any difficulties with the laparoscopic enucleation it is also possible to finish the procedure after exteriorizing the punctured benign lesion directly through the umbilical incision.

The available publications deem ovary-sparing treatment of the affected gonad plausible only in the case of non-cancerous lesions, benign neoplasms and possibly borderline lesions (it increases the risk of recurrence in the case of these neoplasms; however, the recurrences can be efficiently treated without affecting overall survival). Malignant lesions and immature teratomas currently constitute a contraindication to ovary-sparing treatment. In the case of immature teratoma found on the pathology report after sparing surgery it is acceptable to continue the follow up without the absolute necessity for oophorectomy if the tumor markers are negative.

\section{Ovarian biopsy}

This procedure is based on taking a sample of ovarian tissue in order to conduct a histopathological test. The procedure is currently used in the case of suspected pathologies in an ovary during surgical removal of a lesion in the other ovary. There are no studies recommending biopsy when the ovary has a correct macroscopic appearance, and no anomaly is found within the ovary during preoperative imaging tests. Some studies showed a very low percentage of pathological lesion detectability by means of biopsy of the other gonad. There are no studies evaluating the influence of biopsy on the healthy ovary either (only animal studies). That is why biopsy is currently advised only in the case of abnormal appearance of the ovary discovered intraoperatively. However, in the case of suspected dysgerminoma found in a preoperative examination, a biopsy should always be performed. A possibility to perform intraoperative ultrasound 
facilitates the decision-making process concerning a biopsy of the contralateral ovary or even enucleation of a visualized ovary.

\section{Intraoperative histopathological examination}

The most recent guidelines of the American Pediatric Surgical Association recommend intraoperative examination of a frozen sample based on promising results in adults. However, the same data for children are limited. In one study conducted on a small group of pediatric patients, such an examination did not change the initial diagnosis in patients with a large lesion. It was also found that in the case of borderline, large, cystic and mucinous tumors the above-mentioned examination is not reliable.

To summarize, the Polish Association of Pediatric Surgeons does not currently recommend intraoperative histopathological examinations. When a lesion can be enucleated or is limited to an ovary, it should be removed in order to be histopathologically examined. When a lesion proves to be unresectable, a biopsy should be performed, and the surgical procedure should be terminated.

\section{Ethical approval}

The study was approved by the Ethical Committee of the Medical University of Wroclaw - available on request.

\section{Confict of interests}

The authors declare that they have no competing interests.

\section{Funding}

Not applicable

\section{REFERENCES}

1. Gonzalez DO, Minneci PC, Deans KJ. Management of benign ovarian lesions in girls: a trend toward fewer oophorectomies. Curr Opin Obstet Gynecol. 2017; 29(5): 289-294, doi: 10.1097/GCO.0000000000000400, indexed in Pubmed: 28759460.

2. Chae HS, Rheu CH. Precocious pseudopuberty due to an autonomous ovarian follicular cyst: case report with a review of literatures. BMC Res Notes. 2013; 6: 319, doi: 10.1186/1756-0500-6-319, indexed in Pubmed: 23937919.

3. Hermans AJ, Kluivers KB, Janssen LM, et al. Adnexal masses in children, adolescents and women of reproductive age in the Netherlands: A nationwide population-based cohort study. Gynecol Oncol. 2016; 143(1): 93-97, doi: 10.1016/j.ygyno.2016.07.096, indexed in Pubmed: 27421754.

4. Łuczak J, Bagłaj M. Selecting treatment method for ovarian masses in children - 24 years of experience. J Ovarian Res. 2017; 10(1): 59, doi: 10.1186/s13048-017-0353-0, indexed in Pubmed: 28893324.

5. Ehdaivand S WHO classification. PathologyOutlines.com website. https://www.pathologyoutlines.com/topic/ovarytumorwhoclassif. html (2021.04.21)

6. Renaud EJ, Sømme S, Islam S, et al. Ovarian masses in the child and adolescent: An American Pediatric Surgical Association Outcomes and Evidence-Based Practice Committee systematic review. J Pediatr Surg. 2019; 54(3): 369-377, doi: 10.1016/j.jpedsurg.2018.08.058, indexed in Pubmed: 30220452.

7. Brookfield KF, Cheung MC, Koniaris LG, et al. A population-based analysis of 1037 malignant ovarian tumors in the pediatric population. J Surg Res. 2009; 156(1):45-49, doi: 10.1016/j.jss.2009.03.069, indexed in Pubmed: 19592022.
8. Łuczak J, Bagłaj M. Ovarian teratoma in children: a plea for collaborative clinical study.J Ovarian Res. 2018; 11(1): 75, doi: 10.1186/s13048-018-0448-2, indexed in Pubmed: 30165903.

9. Cecchetto G. Gonadal germ cell tumors in children and adolescents. J Indian Assoc Pediatr Surg. 2014; 19(4): 189-194, doi: 10.4103/09719261.141995, indexed in Pubmed: 25336799.

10. Pashankar F, Hale JP, Dang Ha, et al. Is adjuvant chemotherapy indicated in ovarian immature teratomas? A combined data analysis from the Malignant Germ Cell Tumor International Collaborative. Cancer. 2016; 122(2): 230-237, doi: 10.1002/cncr.29732, indexed in Pubmed: 26485622.

11. Park JY, Kim DY, Suh DS, et al. Outcomes of surgery alone and surveillance strategy in young women with stage I malignant ovarian germ cell tumors. Int J Gynecol Cancer. 2016; 26(5): 859-864, doi: 10.1097/IGC.0000000000000702, indexed in Pubmed: 27101582.

12. Stanković ZB, Sedlecky K, Savić D, et al. Ovarian preservation from tumors and torsions in girls: prospective diagnostic study. J Pediatr Adolesc Gynecol. 2017; 30(3): 405-412, doi: 10.1016/j.jpag.2017.01.008, indexed in Pubmed: 28137453.

13. Heo SH, Kim JW, Shin SS, et al. Review of ovarian tumors in children and adolescents: radiologic-pathologic correlation. Radiographics. 2014; 34(7): 2039-2055, doi: 10.1148/rg.347130144, indexed in Pubmed: 25384300.

14. Rekomendacje Sekcji Ultrasonografii Polskiego Towarzystwa Ginekologicznego w zakresie badań ultrasonograficznych w ginekologii. 2015. http://www.usgptg.pl/media/dopobrania/rekomendacje2015/Rekomendacje_ginekologia_2015.pdf (2021.04.21).

15. Kaloo PD, Louden KA, Khazali S, Hoy D, Cheltenham BS, Sadoon S, et al. Management of Suspected Ovarian Masses in Premenopausal Women Green-top Guideline No. 62. Royal College of Obstetricians and Gynaecologists/ British Society for Gynaecological Endoscopy. 2011. https://www. rcog.org.uk/globalassets/documents/guidelines/gtg_62.pdf(2021.04.21).

16. Basta P, Bidzinski M, Kluz T, Nowak-Markwitz E, Olejek A, Sawicki W. Ovarian cancer: recognition and initial management - the recommendation of Polish Gynecological Society. Ginekologia i Perinatologia Praktyczna 2016;1(3):127-129. https://www.ptgin.pl/index.php/lekarze/rekomendacje2/rekomendacje (2021.04.21).

17. Czekierdowski A. Novel ultrasonography and prognostic models in the preoperative discrimination of ovarian cancer. Ginekologia i Perinatologia Praktyczna 2016;1(4:152-161. 2016; 1(4): 152-161.

18. Stankovic ZB, Bjelica A, Djukic MK, et al.Value of ultrasonographic detection of normal ovarian tissue in the differential diagnosis of adnexal masses in pediatric patients. Ultrasound Obstet Gynecol. 2010; 36(1): 88-92, doi: 10.1002/uog.7557, indexed in Pubmed: 20069549.

19. Lam CZ, Chavhan GB. Magnetic resonance imaging of pediatric adnexal masses and mimics. Pediatr Radiol. 2018; 48(9): 1291-1306, doi: 10.1007/s00247-018-4073-4, indexed in Pubmed: 30078037.

20. Al Musalhi K, Al Kindi M, Al Aisary F, et al. Evaluation of HE4, CA-125, risk of ovarian malignancy algorithm (ROMA) and Risk of Malignancy Index (RMI) in the preoperative assessment of patients with adnexal mass. Oman Med J. 2016; 31(5): 336-344, doi: 10.5001/omj.2016.68, indexed in Pubmed: 27602187.

21. Wei SU, Li H, Zhang B. The diagnostic value of serum HE4 and CA-125 and ROMA index in ovarian cancer. Biomed Rep. 2016; 5(1): 41-44, doi: 10.3892/br.2016.682, indexed in Pubmed: 27347403.

22. Romagnolo C, Leon AE, Fabricio ASC, et al. HE4, CA125 and risk of ovarian malignancy algorithm (ROMA) as diagnostic tools for ovarian cancer in patients with a pelvic mass: An Italian multicenter study. Gynecol Oncol. 2016; 141(2): 303-311, doi: 10.1016/j.ygyno.2016.01.016, indexed in Pubmed: 26801941.

23. Sölétormos G, Duffy MJ, Othman Abu Hassan S, et al. Clinical use of cancer biomarkers in epithelial ovarian cancer: updated guidelines from the European Group on Tumor Markers. Int J Gynecol Cancer. 2016; 26(1): 43-51, doi: 10.1097/IGC.0000000000000586, indexed in Pubmed: 26588231.

24. Gonzalez DO, Cooper JN, Aldrink JH, et al.Variability in surgical management of benign ovarian neoplasms in children. J Pediatr Surg. 2017; 52(6): $944-$ 950, doi: 10.1016/j.jpedsurg.2017.03.014, indexed in Pubmed: 28343661.

25. Aldrink JH, Gonzalez DO, Sales SP, et al. Using quality improvement methodology to improve ovarian salvage for benign ovarian masses. J Pediatr Surg. 2017 [Epub ahead of print], doi: 10.1016/j.jpedsurg.2017.10.016, indexed in Pubmed: 29106919.

26. Łuczak J, Bagłaj M, Ciaputa R, et al. Does open ovarian biopsy in prepubertal age affect ovarian reserve in a rat model? J Pediatr Surg. 2021; 56(2): 360 367, doi: 10.1016/j.jpedsurg.2020.05.046, indexed in Pubmed: 32653162. 